\title{
A NEW NONLINEAR $\mathscr{L}$-STABLE SCHEME WITH CONSTANT AND ADAPTIVE STEP-SIZE STRATEGY
}

\author{
Sadia Arain ${ }^{1}$, Sania Qureshi ${ }^{1,2}$, Asif Ali Shaikh ${ }^{1}$ \\ ${ }^{1}$ Basic Sciences and Related Studies, Mehran University of Engineering and Technology \\ Jamshoro, 76062, Sindh, Pakistan \\ ${ }^{2}$ Department of Mathematics, Near East University TRNC, Mersin 10, Turkey \\ sadiatahirmpk@gmail.com, sania.qureshi@faculty.muet.edu.pk, asif.shaikh@faculty.muet.edu.pk
}

Received: 26 October 2021; Accepted: 7 December 2021

\begin{abstract}
The present study proposes a new explicit nonlinear scheme that solves stiff and nonlinear initial value problems in ordinary differential equations. One of the promising features of this scheme is its fourth-order convergence with strong stability having an unbounded region. A modern approach for relative stability growth analysis is also presented under order stars conditions. The scheme is also good in dealing with singular and stiff type of models. Comparing numerical experiments using various errors, including maximum absolute global error over the integration interval, absolute error at the endpoint, average error, norm of errors, and the CPU times (seconds), shows better performance. An adaptive step-size approach seems to increase the performance of the proposed scheme. The numerical simulations assure us of $\mathscr{L}$-stability, consistency, order, and rapid convergence.
\end{abstract}

MSC 2010: 65L05; 65L20; 65L70

Keywords: stiff systems, singular systems, $\mathscr{A}$-stability, local error, order stars

\section{Introduction}

Various real-world applications of natural and physical laws are expressed as mathematical models based upon different types of ordinary differential equations. In [1-4], it has been well explained that the differential equations are useful for understanding the natural and physical world around us with the help of models including mass-spring systems, electrical circuits, population growth, and a few more. It is mostly impossible to get the exact solution of such ordinary differential equations by analytical methods. In such cases, when the analytical method does not work, we try to approximate a solution on the grid points $\left(x_{i}, y_{i}\right), i=1,2,3, \ldots, n$. There are many explicit and implicit numerical techniques to approach the approximate solutions of scalar and dynamical models. In [5-7], authors have mainly discussed applications in engineering, applied mathematics, and other scientific areas, including analysis of numerical methods to improve the order of convergence, the truncation error, stability, and reduction of the computational time. In the derivation of the method discussed 
in [8], derivatives are not involved, and the method solves large systems of ordinary differential equations with reasonable accuracy.

It is described in [9-15] that if initial value problems have singularities and oscillatory solutions, then conventional approaches including single-step Runge-Kutta and linear multistep methods cannot perform well, whereas unconventional approaches can be used to achieve the solution with higher-order numerical accuracy and low computational cost. Such approaches have stronger stability properties as well. Some non-linear or one-step rational schemes in [16,17] or two-step in [18] are applicable for stiff and singular types of non-linear initial value problems. These schemes deal with the singularities at a pole at a given time interval with a low computational cost. The linear RK, non-linear RK, and other such methods do not perform well for a singular type of non-linear equation due to their polynomial nature. These methods do not work well in situations having poles and have some weaker conditions on stability. This is the major drawback of the conventional methods of the RK type. The aim of the proposed non-linear rational scheme is concerned with the convergence order having good stability characteristics. The proposed method uses four evaluations of derivatives with higher computational accuracy. We will first derive the scheme with constant step size and then consider its implementation with adaptive step size in this study.

The paper is arranged as: The formulation of the scheme is developed by a non-linear rational expression in Section 2, while the theoretical analysis is carried out by local error analysis, stability, and order stars in sub-sections. The implementation of the adaptive step-size strategy is available in Section 3. In Section 4, a few experimental results evaluate the new scheme's effectiveness and compare it to schemes of a similar order. Finally, Section 5 gives the conclusion.

\section{Formulation of the proposed scheme}

Consider the following initial value problem of the form:

$$
y^{\prime}(t)=f(t, y(t))
$$

where $y(t) \in \mathbb{R}^{n}$ and $f(t, y(t)): R \times R^{n} \rightarrow R^{n}$ with initial condition $y(a)=y_{0}$ over the interval $t \in[a, b]$. Such types of initial value problems have a unique continuous solution $y(t)$ as per Lipschitz condition as described in [4]. The approximate solution $y_{k}$ of (1) at $t=t_{k}$ is computed that which results in $y_{k} \approx y\left(t_{k}\right)$. The theoretical solution is denoted by $y\left(t_{k}\right)$, where $t_{k}=a+k h$ and $k=0,1, \ldots, N$. The constant grid

size used for this proposed scheme is specified by $h=\frac{b-a}{N}$. Using the ideas from a recently published work [19] where authors have proposed a rational type scheme with third-order convergence, we attempt to formulate a fourth-order scheme to find the approximate solution $y_{k+1}$ of (1). At $t=t_{k+1}$, we imply that the approximate solution is: 


$$
y_{k+1}=\frac{\alpha+\beta h}{1+\gamma h+\phi h^{2}+\theta h^{3}},
$$

where $\alpha, \beta, \gamma, \phi$, and $\theta$ are unknown parameters that would be governed by the known values at $t_{k}$. It may be noted that the denominator in (2) should not be vanished for any value of the parameter. After expanding (2) using the Taylor series, we get the following expression:

$y_{k+1}=\left[\begin{array}{c}\alpha+(\beta-\alpha \gamma) h+\left(-\alpha \phi-\beta \gamma+\alpha \gamma^{2}\right) h^{2}+\left(-\alpha \theta-\beta \phi+2 \alpha \gamma \phi+\beta \gamma^{2}-\alpha \gamma^{3}\right) h^{3} \\ +\left(2 \alpha \gamma \theta-\beta \theta+\alpha \phi^{2}+2 \beta \gamma \phi-3 \alpha \gamma^{2} \phi-\beta \gamma^{3}+\alpha \gamma^{4}\right) h^{4} \\ +\left(-2 \phi \theta-\beta \theta+\alpha^{2} \theta-\beta \phi^{2}+2 \alpha \gamma \phi^{2}+\beta \gamma^{2} \phi-\alpha \gamma^{3} \phi\right) h^{5}\end{array}\right]+\mathscr{O}\left(h^{6}\right)$.

Associating it through the Taylor's series of $y\left(t_{k+1}\right)$ and after comparing the coefficients of the obtained series up to $\mathscr{O}\left(h^{4}\right)$, we will have a non-linear system from which we can easily compute values of unknown parameters as follows:

$$
\begin{aligned}
& \alpha=y_{k}, \\
& \beta=\frac{1}{4}\left(\frac{y k^{i v} y_{k}^{3}+36 y^{\prime \prime}{ }_{k} y_{k}^{\prime 2} y_{k}^{\prime} y_{k}-8 y^{\prime \prime \prime}{ }_{k} y_{k}{ }_{k} y_{k}^{2}-6 y^{\prime \prime 2}{ }_{k} y_{k}^{2}-24 y_{k}^{\prime 4}}{y^{\prime \prime \prime}{ }_{k} y_{k}^{2}-6 y_{k} y^{\prime \prime}{ }_{k} y_{k}^{\prime}+6 y_{k}^{\prime 3}}\right), \\
& \gamma=\frac{1}{4}\left(\frac{-y_{k}^{i v} y_{k}^{2}+4 y_{k} y_{k}^{\prime \prime \prime} y_{k}^{\prime}+6 y_{k}^{\prime \prime^{2}} y_{k}-12 y_{k}^{\prime \prime} y_{k}^{\prime 2}}{y_{k}^{\prime \prime \prime} y_{k}^{2}-6 y_{k} y_{k}^{\prime \prime} y_{k}^{\prime}+6 y_{k}^{\prime 3}}\right), \\
& \phi=\frac{1}{4}\left(\frac{y_{k}^{i v} y_{k}^{\prime} y_{k}-2 y_{k}^{\prime \prime \prime} y_{k}^{\prime \prime} y_{k}-4 y_{k}^{\prime \prime \prime} y_{k}^{\prime 2}+6 y_{k}^{\prime \prime^{2}} y_{k}^{\prime}}{y_{k}^{\prime \prime \prime} y_{k}^{2}-6 y_{k} y_{k}^{\prime \prime} y_{k}^{\prime}+6 y_{k}^{\prime 3}}\right), \\
& \theta=\frac{1}{24}\left(\frac{3 y_{k}^{i v} y_{k}^{\prime \prime} y_{k}-4 y_{k}^{\prime \prime \prime^{2}}-6 y_{k}^{i v} y_{k}^{\prime 2}+24 y_{k}^{\prime \prime \prime} y_{k}^{\prime \prime} y_{k}^{\prime}-18 y_{k}^{\prime \prime^{3}}}{y_{k}^{\prime \prime \prime} y_{k}^{2}-6 y_{k} y_{k}^{\prime \prime} y_{k}^{\prime}+6 y_{k}^{\prime 3}}\right) .
\end{aligned}
$$

Substituting the values of these parameters in (2) and after simplifying it further, we get the nonlinear scheme with fourth-order convergence as shown below:

$$
\begin{gathered}
y_{k+1}=\frac{6\left(\left(4\left(y_{k}+2 h f_{k}\right) j_{k} y_{k}^{2}-\left(l_{k} y_{k}-6 g_{k}^{2}\right) h y_{k}^{2}-12\left(3 h f_{k}+2 y_{k}\right) g_{k} f_{k} y_{k}+24\left(h f_{k}+y_{k}\right) f_{k}^{3}\right)\right.}{3\left(h^{2} g_{k} y_{k}-2 h^{2} f_{k}^{2}+2 h f_{k} y_{k}-2 y_{k}^{2}\right) h l_{k}-4 h^{3} j_{k}^{2} y_{k}+12\left(\left(2 h f_{k}-y_{k}\right) h^{2} g_{k}-2 h^{2} f_{k}^{2}\right.} \\
\left.+2 h f_{k} y_{k}+2 y_{k}^{2}\right) j_{k}-18 h^{3} g_{k}^{3}+36 h\left(h f_{k}+y_{k}\right) g_{k}^{2}-72\left(\left(h f_{k}+2 y_{k}\right) g_{k}+2 f_{k}^{2}\right) f_{k}
\end{gathered}
$$


where $y_{k}=y\left(t_{k}\right), y_{k+1}=y\left(t_{k+1}\right), f_{k}=f\left(t_{k}, y_{k}\right), g_{k}=\frac{d}{d t} f\left(t_{k}, y_{k}\right), j_{k}=\frac{d^{2}}{d t^{2}} f\left(t_{k}, y_{k}\right)$, and $l_{k}=\frac{d^{3}}{d t^{3}} f\left(t_{k}, y_{k}\right)$. It may be noted that the scheme requires 4 derivatives prior to starting the iterations. This approach makes the scheme stronger than several existing approaches.

\subsection{Theoretical analysis}

In this section, the proposed fourth-order scheme given in (9) is analyzed theoretically.

\subsubsection{Local error analysis}

The investigation of the local truncation error determines the order of convergence for any numerical technique designed to solve initial value problems. Firstly, to find the order of consistency of the newly formulated scheme, we define the local truncation error as:

$$
L(u(t), h)=u(t+h)-u\left(t_{k+1}\right)
$$

where $u(t)$ is an arbitrary function specified over the integration interval $[a, b]$, we can differentiate it as many times as we require. Expanding (10) via Taylor's series, the scheme's local truncation error can be described as:

$L T E_{n+1}=\frac{1}{P}\left[\begin{array}{c}4 y_{k}^{i v} y_{k}^{\prime \prime \prime} y_{k}^{2}-5 y_{k}^{i v} y_{k}^{2}-24 y_{k}^{v} y_{k}^{\prime \prime} y_{k}^{\prime} y_{k}+60 y_{k}^{i v} y_{k}^{\prime \prime 2} y_{k}-80 y_{k}^{\prime \prime \prime 2} y_{k}^{2} \\ +24 y_{k}^{v} y_{k}^{\prime 3}-120 y_{k}^{i v} y_{k}^{\prime \prime} y_{k}^{\prime 2}-80 y_{k}^{\prime \prime \prime 2} y_{k}{ }_{k}+360 y_{k}^{\prime \prime \prime}{ }_{k} y_{k}^{\prime \prime 2} y_{k}^{\prime}-80 y_{k}{ }_{k} 4\end{array}\right] h^{5}+\mathscr{O}\left(h^{6}\right)$,

where $P=480 y^{\prime \prime}{ }_{k} y_{k}^{2}+2880\left(y_{k}^{\prime 2}-y_{k}^{\prime \prime} y_{k}\right) y_{k}^{\prime} \neq 0$. The notations $y_{k}^{\prime}, y_{k}^{\prime \prime}, y_{k}^{\prime \prime \prime}, y_{k}^{(i v)}$, and $y_{k}^{(v)}$ indicate the first-, second-, third-, fourth- and fifth-order derivatives of $y(t)$ computed at the point $t_{k}$.

\subsubsection{Stability analysis, order stars and consistency}

The Dahlquist's model given below:

$$
y^{\prime}=\lambda y, \operatorname{Re}(\lambda)<0,
$$

is always used to analyze stability of a newly formulated scheme for initial value problems. When the proposed nonlinear scheme (9) is employed on (12), then 
the following difference equation is achieved:

$$
y_{n+1}=-\frac{6(h \lambda+4)}{h^{3} \lambda^{3}-6 h^{2} \lambda^{2}+18 h \lambda-24} y_{n} .
$$

Setting $z=h \lambda$, the rational stability function is written as follows:

$$
\Phi(z)=-\frac{6(z+4)}{z^{3}-6 z^{2}+18 z-24},
$$

and the region of absolute stability is defined as:

$$
R=\{z \in \mathbb{C}:|\Phi(z)|<1\}
$$

The region of absolute stability of the proposed nonlinear scheme (9), as shown in Figure 1, consists of the entire left half complex plane, which means that the scheme has characteristics of being $\mathscr{A}$-stable (see [2]) while its $3 \mathrm{D}$ graphic surface is also obtained in Figure 2. In addition, the scheme satisfies the condition $\lim _{z \rightarrow-\infty} \Phi(z)=0$ which means that the proposed scheme is not only $\mathscr{A}$-stable but $\mathscr{L}$-stable as well. Furthermore, since the scheme has at least a fourth-order of accuracy, it therefore also satisfies the condition of consistency. Finally, the order stars capture a more chaotic pattern by showing the detection of all poles, zeros, and interpolation points as shown in Figure 3.

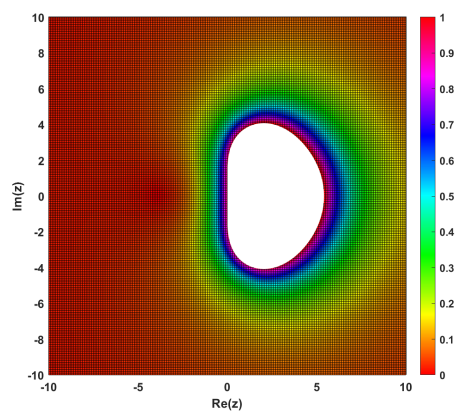

Fig. 1. Stability region (shaded) of the proposed fourth order rational scheme

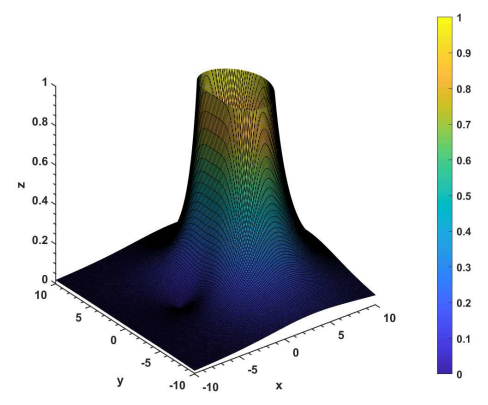

Fig. 2. 3D graphical surface view of the proposed fourth order nonlinear scheme 


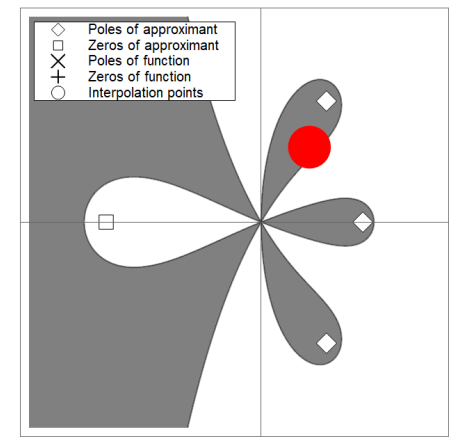

Fig. 3. The placement of order stars emerging from the stability rational function of the proposed scheme

\section{Adaptive step-size strategy}

The newly proposed scheme in (9) has been formulated with a constant stepsize $h$ in the previous section. Nevertheless, as several authors such as [20] have pointed out, a numerical integrator must be more suitable for an adaptive step-size formulation that is based on an explicit formula.

Now, the proposed scheme is discussed in adaptive step-size form by using a lower order approach to create a strategy to estimate the local error $\left(L E_{n}\right)$ at the end-point on the interval. The cost of the computational process will be less in this case. We used a similar embedded type approach which has been used in [19] as given below:

$$
y_{k+1}=\frac{2\left(6 f_{k}^{3}+6 f_{k}\left(f_{k}-h j_{k}\right) y_{k}+\left(h k_{k}-3 j_{k}\right) y_{k}^{2}\right)}{12 f_{k}^{2}+3 h^{2} f_{k}^{2}-f_{k} h\left(h k_{k}+3 j_{k}\right)+\left(2 h k_{k}-6 j_{k}\right) y_{k}} .
$$

Firstly, we want to get an acceptable estimate value of $L E_{n}$ by a lower order scheme. We will follow the same strategy as described in [1]. Now, consider:

$$
L E_{n}=y\left(t_{k}+h\right)-y_{k+1} \text {. }
$$

The local error can be obtained for $y_{k+1}$ by using a scheme of order $p$, whereas for $y_{k+1}^{*}$, we can obtain it by using a strategy of order $p+1$ as shown below:

$$
\begin{gathered}
E S T=\left\{y\left(t_{k}+h\right)-y_{k+1}\right\}-\left\{y_{k+1}^{*}-y\left(t_{k}+h\right)\right\} \\
=L E_{n}-\mathscr{O}\left(h^{p+2}\right) .
\end{gathered}
$$

The local error $L E_{n}$ is of order $\mathscr{O}\left(h^{p+1}\right)$, therefore, the error dominates in (16) for small values of $h$. This is a computational local error estimation of the lower-order formula. We can estimate the error in $y_{k+1}$ by comparing it to the more accurate approximate solution of $y_{k+1}^{*}$. This is a way to find a pair of embedded type of scheme that shares as many functions evaluations as possible. Local extrapolation is the technique of advancing the integration using the more accurate result in $y_{k+1}^{*}$. 
The step would be rejected if the estimated error is too large compared to the given local error tolerance $(\tau)$, and then it further tries to take a smaller step size. We only worked out the order of the first nonzero term in our expansion of the local error. If another term is included in the expansion, then we get the following:

$$
y\left(t_{k}+h\right)-y_{k+1}=h^{p+1} \varphi\left(t_{k}\right)+\mathscr{O}\left(h^{p+2}\right) .
$$

If we take a step from $t_{k}$ with a new step size $\rho h$, we will reach at the following step:

$$
\begin{gathered}
(\rho h)^{p+1} \sigma\left(t_{k}\right)+ \\
\mathscr{O}(\rho h)^{p+2}=\rho^{p+1} h^{p+1} \sigma\left(t_{k}\right)+\mathscr{O}\left(h^{p+2}\right) \\
=\rho^{p+1} E S T+\mathscr{O}\left(h^{p+2}\right) .
\end{gathered}
$$

The largest step size that we predict will pass the error test corresponding to $\rho$ so that:

$$
\left\|\rho^{p+1} E S T\right\| \approx \tau
$$

The new step-size is decided as follows:

$$
h_{\text {new }}=h_{\text {old }}\left[\frac{\tau}{\|E S T\|}\right]^{\frac{1}{p+1}} .
$$

If the local error is smaller than the given tolerance, it is necessary to increase step size $h$. Larger step sizes reach the end of the integration interval in fewer steps, making the computation more efficient. However, the step size is estimated using several approximations. A failed step is always costly. The algorithm (19) uses a fraction $(\eta)$ of the expected step size as shown below:

$$
h_{\text {new }}=\eta h_{\text {old }}\left[\frac{\tau}{\|E S T\|}\right]^{\frac{1}{p+1}} .
$$

Here, $p=3$ denotes the order of the lower order scheme, and $0<\eta<1$ denotes a reasonable adjustment factor for avoiding unsuccessful steps. We use $\eta=0.9$ in our numerical experiments.

\section{Numerical experiments}

Various numerical experiments have been carried out to test the performance of the proposed scheme given in (9). The comparison is made with the fourth-order Taylor series and a nonlinear RK type scheme based upon harmonic mean (RK-HM) [21]. The accuracy is measured by using the following error formulas: Maximum absolute error: $E_{\max }=\max \left\|y\left(t_{k}\right)-y_{k}\right\|$, Absolute error at the end point $t=b$ : $E_{t_{t=b}}=\left\|y(b)-y_{k}\right\|$, Average error: $E_{A}=\frac{1}{N} \sum_{k=0}^{N} y_{k}$, error norm: $E_{\text {norm }}=\sqrt{\sum_{k=0}^{N}\left\|y_{k}\right\|^{2}}$ 
and CPU time measured in seconds. Here $N$ is the total number of sub-intervals. The theoretical and numerical solutions of a test problem at point $t_{k}$ are represented by $y\left(t_{k}\right)$ and $y_{k}$, respectively. MAT LAB software having version '9.2.0.538062 $(R 2017 a)^{\prime}$ will be used for numerical simulations. Here, NI stands for the number of iterations.

\subsection{Example 1}

Consider a non-autonomous initial value problem [19]:

$$
y^{\prime}(t)=(\lambda-1) e^{-\lambda t}-y(t), \lambda=500,
$$

and with initial condition $y(0)=1$ over interval $[0,1]$ with $y(t)=2 e^{-t}-e^{-\lambda t}$. The proposed scheme in (9) was applied to this problem, and the results of different errors are given in Table 1 by using different numbers of step size values. In this problem, the performance of (9) is better than Taylor and RK-HM. It is observed in Table 1 that the errors are decreased with various constant step sizes. When we apply the adaptive step size approach with different tolerances with $h=0.1$, the new scheme reduces the steps as shown in Table 2.

Table 1. The absolute error at the endpoint in the first row, maximum absolute error in the second row, the average error in the third row, the norm in the fourth row, and CPU time in the fifth row with constant step-size approach on the integration interval $[0,1]$ for the IVP (20)

\begin{tabular}{c|ccccc}
\hline Scheme/NI & 128 & 256 & 512 & 1024 & 2048 \\
\hline \multirow{5}{*}{ Taylor } & $1.6917 \mathrm{e}+00$ & $7.6205 \mathrm{e}-02$ & $3.7569 \mathrm{e}-03$ & $2.0402 \mathrm{e}-04$ & $1.1819 \mathrm{e}-05$ \\
& $4.5253 \mathrm{e}+00$ & $2.0414 \mathrm{e}-01$ & $1.0064 \mathrm{e}-02$ & $5.4662 \mathrm{e}-04$ & $3.1665 \mathrm{e}-05$ \\
& $2.8722 \mathrm{e}+00$ & $1.3004 \mathrm{e}-01$ & $6.4245 \mathrm{e}-03$ & $3.4919 \mathrm{e}-04$ & $2.0236 \mathrm{e}-05$ \\
& $3.4062 \mathrm{e}+01$ & $2.1719 \mathrm{e}+00$ & $1.5143 \mathrm{e}-01$ & $1.1629 \mathrm{e}-02$ & $9.5270 \mathrm{e}-04$ \\
& $1.8657 \mathrm{e}-02$ & $1.4480 \mathrm{e}-02$ & $2.5393 \mathrm{e}-02$ & $1.4602 \mathrm{e}-02$ & $7.2115 \mathrm{e}-02$ \\
\hline \multirow{5}{*}{ RK-HM } & $1.6328 \mathrm{e}-01$ & $5.4077 \mathrm{e}-02$ & $1.3742 \mathrm{e}-02$ & $3.7686 \mathrm{e}-03$ & $9.1029 \mathrm{e}-04$ \\
& $4.3700 \mathrm{e}-01$ & $1.4563 \mathrm{e}-01$ & $3.8866 \mathrm{e}-02$ & $1.0146 \mathrm{e}-02$ & $2.5126 \mathrm{e}-03$ \\
& $2.7721 \mathrm{e}-01$ & $9.2272 \mathrm{e}-02$ & $2.3516 \mathrm{e}-02$ & $6.4483 \mathrm{e} 03$ & $1.5590 \mathrm{e}-03$ \\
& $3.2876 \mathrm{e}+00$ & $1.5410 \mathrm{e}+00$ & $5.5444 \mathrm{e}-01$ & $2.1473 \mathrm{e}-01$ & $7.3403 \mathrm{e}-02$ \\
& $2.2657 \mathrm{e}-02$ & $2.2280 \mathrm{e}-02$ & $3.4330 \mathrm{e}-02$ & $6.9855 \mathrm{e}-02$ & $6.3042 \mathrm{e}-02$ \\
\hline \multirow{5}{*}{ Proposed } & $1.2163 \mathrm{e}-00$ & $1.2595 \mathrm{e}-03$ & $8.6301 \mathrm{e}-05$ & $5.0510 \mathrm{e}-06$ & $2.8597 \mathrm{e}-07$ \\
& $3.8659 \mathrm{e}-02$ & $5.3299 \mathrm{e}-03$ & $3.8887 \mathrm{e}-04$ & $2.6225 \mathrm{e}-05$ & $1.6693 \mathrm{e}-06$ \\
& $2.0703 \mathrm{e}-02$ & $2.1611 \mathrm{e}-03$ & $1.4869 \mathrm{e}-04$ & $8.7267 \mathrm{e}-06$ & $4.9509 \mathrm{e}-07$ \\
& $2.4587 \mathrm{e}-01$ & $3.6234 \mathrm{e}-02$ & $3.5228 \mathrm{e}-03$ & $2.9259 \mathrm{e}-04$ & $2.3512 \mathrm{e}-05$ \\
& $3.4330 \mathrm{e}-02$ & $1.8204 \mathrm{e}-02$ & $3.2943 \mathrm{e} 02$ & $2.8086 \mathrm{e}-02$ & $3.4555 \mathrm{e}-02$ \\
\hline
\end{tabular}

Table 2. The number of steps $n$ in the first row and the final absolute error with adaptive step-size approach on the integration interval $[0,0.5]$ for the IVP (20)

\begin{tabular}{c|cccc}
\hline Scheme/Tolerance & $10^{-1}$ & $10^{-2}$ & $10^{-3}$ & $10^{-4}$ \\
\hline \multirow{2}{*}{ Taylor } & 9 & 10 & 13 & 18 \\
& $6.286 \mathrm{e}-02$ & $1.3848 \mathrm{e}-02$ & $4.4411 \mathrm{e}-03$ & $9.5161 \mathrm{e}-04$ \\
\hline \multirow{2}{*}{ RK-HM } & 8 & 10 & 18 & 33 \\
& $3.0747 \mathrm{e}-02$ & $1.2038 \mathrm{e}-02$ & $4.4411 \mathrm{e}-03$ & $9.1439 \mathrm{e}-04$ \\
\hline \multirow{2}{*}{ Proposed } & 8 & 10 & 13 & 20 \\
& $5.4731 \mathrm{e}-03$ & $6.0627 \mathrm{e}-04$ & $1.1896 \mathrm{e}-04$ & $1.8482 \mathrm{e}-05$ \\
\hline
\end{tabular}




\subsection{Example 2}

Consider a Bernoulli differential equation.

$$
t y^{\prime}=y^{2} \ln (t)-y,
$$

and with initial condition $y(0.1)=-2$ at interval $[0.1,1]$ which has exact solution

$$
y(t)=\frac{1}{1-15 t+10 t \ln (10)+\ln (t)} .
$$

In Table 3, we can observe that the Taylor series and RK-HM could not solve this problem and fail before the singularity, whereas the new scheme is capable of solving that issue. The adaptive step size implementation is represented by Table 4. As can be seen, the proposed strategy took quite a few steps to achieve an accurate result.

Table 3. The absolute error at the endpoint in the first row, maximum absolute error in the second row, the average error in the third row, the norm in the fourth row, and CPU time in the fifth row with a constant step-size approach on the integration interval $[0.1,1]$ for the IVP (21)

\begin{tabular}{c|ccccc}
\hline Scheme/NI & 128 & 256 & 512 & 1024 & 2048 \\
\hline Taylor & div & div & div & div & div \\
\hline RK-HM & div & div & div & div & div \\
\hline & $7.4302 \mathrm{e}-09$ & $4.6697 \mathrm{e}-10$ & $1.1715 \mathrm{e}-09$ & $3.7251 \mathrm{e}-10$ & $1.4985 \mathrm{e}-11$ \\
& $7.8761 \mathrm{e}-05$ & $4.9536 \mathrm{e}-06$ & $1.3352 \mathrm{e}-04$ & $1.7389 \mathrm{e}-05$ & $2.3385 \mathrm{e}-06$ \\
Proposed & $7.5837 \mathrm{e}-07$ & $4.5842 \mathrm{e}-08$ & $3.1431 \mathrm{e}-07$ & $3.8293 \mathrm{e}-08$ & $3.0519 \mathrm{e}-09$ \\
& $7.9359 \mathrm{e}-05$ & $6.7368 \mathrm{e}-06$ & $1.3445 \mathrm{e}-04$ & $1.9814 \mathrm{e}-05$ & $3.082 \mathrm{e}-06$ \\
& $2.0781 \mathrm{e}-02$ & $2.7003 \mathrm{e}-02$ & $2.7643 \mathrm{e} 02$ & $3.0142 \mathrm{e}-02$ & $5.3498 \mathrm{e}-02$ \\
\hline
\end{tabular}

Table 4. The number of steps $n$ in the first row and the final absolute error with adaptive step-size approach on the integration interval $[0.1,1]$ for the IVP $(21)$

\begin{tabular}{c|cccc}
\hline Scheme/Tolerance & $10^{-1}$ & $10^{-2}$ & $10^{-3}$ & $10^{-4}$ \\
\hline Taylor & $\operatorname{div}$ & $\operatorname{div}$ & $\operatorname{div}$ & $\operatorname{div}$ \\
\hline RK-HM & $\operatorname{div}$ & $\operatorname{div}$ & $\operatorname{div}$ & $\operatorname{div}$ \\
\hline \multirow{2}{*}{ Proposed } & 5 & 6 & 11 & 16 \\
& $3.2771 \mathrm{e}-04$ & $6.6587 \mathrm{e}-05$ & $1.7878 \mathrm{e}-05$ & $3.4765 \mathrm{e}-06$ \\
\hline
\end{tabular}

\subsection{Example 3}

Consider an autonomous non-linear initial value problem $[18,20]$ having singularity at $t=\pi / 4$ :

$$
y^{\prime}(t)=1+y^{2}, y(0)=1, t \in[0,1],
$$

with exact solution $y(t)=\tan (t+\pi / 4)$. In this problem, Table 5 proved that a fourthorder Taylor series and RK-HM schemes could not solve this problem at $\frac{\pi}{4}$, and the proposed scheme gives better results and it crosses the singularity without any difficulty. Furthermore, Table 6 has shown that the adaptive step-size approach reduces 
the error at the endpoint of the interval and takes fewer steps in the proposed scheme in comparison.

Table 5. The absolute error at the endpoint in the first row, maximum absolute error in the second row, the average error in the third row, the norm in the fourth row, and CPU time in the fifth row with a constant step-size approach on the integration interval $[0,1]$ for the IVP 22

\begin{tabular}{c|ccccc}
\hline Scheme/NI & 32 & 64 & 128 & 256 & 512 \\
\hline Taylor & div & div & div & div & div \\
\hline RK-HM & div & div & div & div & div \\
\hline & $2.1147 \mathrm{e}-06$ & $1.3319 \mathrm{e}-07$ & $8.3983 \mathrm{e}-09$ & $4.6076 \mathrm{e}-07$ & $4.2398 \mathrm{e}-08$ \\
& $4.0092 \mathrm{e}-03$ & $2.5356 \mathrm{e}-04$ & $2.0774 \mathrm{e}-05$ & $1.5567 \mathrm{e}-03$ & $6.5608 \mathrm{e}-04$ \\
Proposed & $1.2839 \mathrm{e}-04$ & $4.9278 \mathrm{e}-06$ & $3.4837 \mathrm{e}-07$ & $1.0736 \mathrm{e}-05$ & $2.0004 \mathrm{e}-06$ \\
& $4.0109 \mathrm{e}-03$ & $2.5618 \mathrm{e}-04$ & $2.6369 \mathrm{e}-05$ & $1.6401 \mathrm{e}-03$ & $6.7619 \mathrm{e}-04$ \\
& $1.6146 \mathrm{e}-02$ & $1.9831 \mathrm{e}-02$ & $2.0672 \mathrm{e}-02$ & $1.7373 \mathrm{e}-02$ & $1.8292 \mathrm{e}-02$ \\
\hline
\end{tabular}

Table 6. The number of steps $n$ in the first row and the final absolute error with adaptive step-size approach on the integration interval $[0,0.5]$ for the IVP 22

\begin{tabular}{c|cccc}
\hline Scheme/Tolerance & $10^{-1}$ & $10^{-2}$ & $10^{-3}$ & $10^{-4}$ \\
\hline \multirow{2}{*}{ Taylor } & 3 & 4 & 6 & 9 \\
& $1.1613 \mathrm{e}-01$ & $2.7449 \mathrm{e}-02$ & $6.2407 \mathrm{e}-03$ & $1.5063 \mathrm{e}-03$ \\
\hline \multirow{2}{*}{ RK-HM } & 3 & 4 & 5 & 8 \\
& $1.0594 \mathrm{e}-01$ & $1.8345 \mathrm{e}-02$ & $9.2097 \mathrm{e}-03$ & $8.644 \mathrm{e}-04$ \\
\hline \multirow{2}{*}{ Proposed } & 2 & 3 & 4 & 7 \\
& $1.6783 \mathrm{e}-02$ & $2.4315 \mathrm{e}-03$ & $4.1979 \mathrm{e}-04$ & $5.0274 \mathrm{e}-05$ \\
\hline
\end{tabular}

\section{Conclusion}

We have constructed a new nonlinear explicit scheme in this paper that effectively solves initial value problems in ordinary differential equations. The scheme is fourth-order convergent, consistent, and $\mathscr{L}$-stable. Due to $\mathscr{L}$-stability property, the scheme is more efficient for solving singular and stiff models. The numerical results produced by the proposed scheme for the problems under consideration show that it improves some existing approaches. The adaptive step-size formulation of the proposed scheme also improves its efficiency as far as the number of steps is concerned. For future studies, the most dynamic field of numerical fractional calculus, as described in [22-24], will be taken into consideration, and the new numerical schemes will be proposed. 


\section{References}

[1] Shampine, L.F., Gladwell, I., Shampine, L., \& Thompson, S. (2003). Solving ODEs with Matlab. Cambridge University Press.

[2] Hairer, E., \& Wanner, G. (1991). Solving Ordinary Differential Equations II. Berlin-Heidelberg: Springer.

[3] Asif, M., Jan, S.U., Haider, N., Al-Mdallal, Q., \& Abdeljawad, T. (2020). Numerical modeling of npz and sir models with and without diffusion. Results in Physics, 19, 103512.

[4] Stoer, J., \& Bulirsch, R. (2013). Introduction to Numerical Analysis. (Vol. 12). Springer Science $\&$ Business Media.

[5] Qureshi, S., \& Yusuf, A. (2020). A new third-order convergent numerical solver for continuous dynamical systems. Journal of King Saud University-Science, 32(2), 1409-1416.

[6] Emmanuel, F.S., \& Qureshi, S. (2019). Convergent numerical method using the transcendental function of exponential type to solve continuous dynamical systems. Journal of Mathematics, 51(10), 45-56.

[7] Gadella, M., \& Lara, L.P. (2013). A numerical method for solving ODE by rational approximation. Applied Mathematical Sciences, 7(23), 1119-1130.

[8] Sharma, J.R., Arora, H., \& Petković, M.S. (2014). An efficient derivative-free family of fourthorder methods for solving systems of nonlinear equations. Applied Mathematics and Computation, 235, 383-393.

[9] Qureshi, S., Memon, Z., \& Shaikh, A.A. (2018). Local accuracy and error bounds of the improved Runge-Kutta numerical methods. Journal of Applied Mathematics and Computational Mechanics, 17(4).

[10] Memon, Z., Qureshi, S., Memon, B.R., \& Saleem, M. (2019). An optimized single-step method for integrating Cauchy problems. Journal of Mathematics, 51(9), 33-44.

[11] Yaakub, A.R., \& Evans, D.J. (1999). A fourth order Runge-Kutta RK $(4,4)$ method with error control. International Journal of Computer Mathematics, 71(3), 383-411.

[12] Awoyemi, D.O. (2005). Algorithmic collocation approach for direct solution of fourth-order initial-value problems of ordinary differential equations. International Journal of Computer Mathematics, 82(3), 321-329.

[13] Qureshi, S., Soomro, A., \& Hınçal, E. (2021). A new family of a-acceptable nonlinear methods with fixed and variable stepsize approach. Computational and Mathematical Methods, e1213.

[14] Owolabi, K.M. (2011). The 4th-step implicit formula for the solution of initial value problems of second-order ordinary differential equations. African Journal of Mathematics and Computer Science Research, 4(7), 270-272.

[15] Ramos, H., Qureshi, S., \& Soomro, A. (2021). Adaptive step-size approach for Simpson's-type block methods with time efficiency and order stars. Computational and Applied Mathematics, 40(6), 1-20.

[16] Ramos, H. (2007). A Nonlinear Explicit One-Step Integration Scheme for Singular Autonomous Initial Value Problems. In AIP Conference Proceedings (Vol. 936, No. 1, pp. 448-451). American Institute of Physics.

[17] Ramos, H., Singh, G., Kanwar, V., \& Bhatia, S. (2015). Solving first-order initial-value problems by using an explicit non-standard A-stable one-step method in the variable step-size formulation. Applied Mathematics and Computation, 268, 796-805.

[18] Ying, T.Y. (2014). An explicit two-step rational method for the numerical solution of first order initial value problem. In AIP Conference Proceedings (Vol. 1605, No. 1, pp. 96-100). American Institute of Physics. 
[19] Qureshi, S., \& Ramos, H. (2018). The L-stable explicit nonlinear method with constant and variable step-size formulation for solving initial value problems. International Journal of Nonlinear Sciences and Numerical Simulation, 19(7-8), 741-751.

[20] Ramos, H., Singh, G., Kanwar, V., \& Bhatia, S. (2017). An embedded 3(2) pair of nonlinear methods for solving first order initial-value ordinary differential systems. Numerical Algorithms, 75(3), 509-529.

[21] Sanugi, B.B., \& Evans, D.J. (1994). A new fourth order Runge-Kutta formula based on the harmonic mean. International Journal of Computer Mathematics, 50(1-2), 113-118.

[22] Abdeljawad, T., Amin, R., Shah, K., Al-Mdallal, Q., \& Jarad, F. (2020). Efficient sustainable algorithm for numerical solutions of systems of fractional order differential equations by Haar wavelet collocation method. Alexandria Engineering Journal, 59(4), 2391-2400.

[23] Abdeljawad, T., Hajji, M.A., Al-Mdallal, Q.M., \& Jarad, F. (2020). Analysis of some generalized abc-fractional logistic models. Alexandria Engineering Journal, 59(4), 2141-2148.

[24] Al-Mdallal, Q.M., Hajji, M.A., \& Abdeljawad, T. (2021). On the iterative methods for solving fractional initial value problems: new perspective. Journal of Fractional Calculus and Nonlinear Systems, 2(1), 76-81. 